\title{
CARACTERIZACIÓN QUÍMICA DEL MATERIAL PARTICULADO ATMOSFÉRICO DEL CENTRO URBANO DE HUANCAYO, PERÚ
}

\author{
Luis Suárez-Salas ${ }^{* a}$, Daniel Álvarez Tolentino ${ }^{a}$, Yéssica Bendezúb ${ }^{\text {, José Pomalaya }}{ }^{\mathrm{b}}$
}

\begin{abstract}
RESUMEN
La ciudad de Huancayo, ubicada en los Andes centrales del Perú, presenta problemas de calidad del aire, siendo el principal contaminante el material particulado (MP). Por ello, el objetivo del presente artículo fue caracterizar la composición química del MP atmosférico colectados en una estación ubicada en el centro urbano de Huancayo. Se colectó MP en agosto del 2007, y enero, abril y mayo del 2008 con un equipo de bajo volumen (PARTISOL FRM 2000) y filtros de $47 \mathrm{~mm}$. Se determinó la concentración de partículas de tamaño hasta $10 \mu \mathrm{m}\left(\mathrm{MP}_{10}\right)$, partículas menores a 2,5 $\mu \mathrm{m}\left(\mathrm{MP}_{2,5}\right)$ y se analizó la composición química de hasta 38 elementos, mediante la técnica de fluorescencia de rayos X (XRF). Y en el caso del $\mathrm{MP}_{2.5}$ se analizó los iones: nitrato $\left(\mathrm{NO}_{3}^{-}\right)$y sulfato $\left(\mathrm{SO}_{4}{ }^{-}\right)$y especies carbonáceas: carbono orgánico (COrg) y carbono elemental (CE).

Los resultados de $\mathrm{MP}_{10}\left(64,54 \pm 30,87 \mu \mathrm{g} / \mathrm{m}^{3}\right)$ y $\mathrm{MP}_{2,5}\left(34,47 \pm 14,75 \mu \mathrm{g} / \mathrm{m}^{3}\right)$ superaron la normativa anual de calidad del aire peruano, siendo el $\mathrm{MP}_{2,5}$ el que representa mayor abundancia en comparación a otras ciudades importantes. También se evidencia una mayor concentración de $\mathrm{MP}_{10} \mathrm{y} \mathrm{MP}_{2,5}$ en periodo seco. La determinación de la composición química indicó que los elementos geológicos $(8 \%)$, metales $(12 \%)$ y metaloides $(7 \%)$ fueron los componentes más abundantes en el $\mathrm{MP}_{10}$ y los no metales (4\%), iones (11\%) y especies carbonáceas $(44 \%)$ en el $\mathrm{MP}_{2,5}$. En conclusión este estudio confirma la problemática del MP en la ciudad de Huancayo, pero evidencia el mayor riesgo del $\mathrm{MP}_{2,5}$ a la salud de su población, siendo el sector transporte una de las fuentes de emisión más importantes.
\end{abstract}

Palabras clave: Composición química, material particulado, Huancayo.

\section{CHEMICAL CHARACTERIZATION OF PARTICULATE MATTER AT AN URBAN SITE OF HUANCAYO CITY, PERU}

\begin{abstract}
Huancayo city, located at the Central Andes of Peru, presents air quality problems being the main pollutant the particulate matter (PM). For that reason, the objective of this paper was to characterize the chemical composition of atmospheric PM sampled in a station located
\end{abstract}

\footnotetext{
a Observatorio de Huancayo, Instituto Geofísico del Perú. Email: 1suarez@igp.gob.pe
}

${ }^{\text {b }}$ Facultad de Ingeniería Química de la Universidad Nacional del Centro del Perú. 
in urban center of Huancayo city. It was sampled PM in august 2007, and January, April and May 2008 with a low volume sampler (Partisol FRM2000) and $47 \mathrm{~mm}$ filters. It was determined the concentration of particles smaller than $10 \mathrm{um}$, particles smaller than 2.5 um and it was analyzed the chemical composition up to 38 elements, using the X Rays Fluorescence (XRF) technique. In the case of $(\mathrm{MP} 2,5)$ additional analysis were performed for ionic composition: nitrates $\left(\mathrm{NO}_{3}^{-}\right)$, sulfates $\left(\mathrm{SO}_{4}{ }^{-}\right)$and carbonaceous species: organic carbon (COrg) and elemental carbon (CE).

The results of $\mathrm{MP}_{10}\left(64.54 \pm 30.87 \mu \mathrm{g} / \mathrm{m}^{3}\right)$ y $\mathrm{MP}_{2.5}\left(34.47 \pm 14.75 \mu \mathrm{g} / \mathrm{m}^{3}\right)$ were higher that the annual Peruvian air quality standard, being the MP2,5 the one that registered higher values than other important cities. Also higher values of $\mathrm{MP}_{10} \mathrm{y} \mathrm{MP}_{2,5}$ were registered during the dry season. The determination of the chemical composition showed that geological elements $(8 \%)$, metals $(12 \%)$ and metalloids $(7 \%)$ were the most abundant elements in the MP10 and the non-metals (4\%), ions $(11 \%)$ and carbonaceous species $(44 \%)$ in the $\mathrm{MP}_{2,5}$. In conclusion this study confirms the problem of PM in Huancayo city, but provides important evidence the higher risk of $\mathrm{MP}_{2,5}$ to the health of its population, being the transport sector on of the most important emission sources.

Key words: Chemical composition, particulate matter, Huancayo city.

\section{INTRODUCCIÓN}

El material particulado (MP) atmosférico es una compleja mezcla de partículas sólidas y líquidas presentes en el aire y su composición química representa una complejidad aún mayor. Para un mejor estudio, es común la medición de las fracciones del material particulado: partículas menores de 10 micrómetros $(\mu \mathrm{m}), \mathrm{MP}_{10}$ y partículas menores de $2,5 \mu \mathrm{m}, \mathrm{MP}_{2,5}$, las cuales varían ampliamente con respecto a su concentración y composición química según el tiempo y lugar. Según su origen se clasifican en partículas primarias, provenientes de fuentes directas como: la quema de biomasa, combustión incompleta de combustibles fósiles, erupciones volcánicas, desgaste de carreteras, suelo, mar y materiales biológicos (fragmentos de plantas, microorganismos, polen, etc.); y partículas secundarias, provenientes principalmente de la conversión de gases a partículas en la atmosfera ${ }^{1}$.

El MP tiene una profunda influencia en la salud pública, visibilidad atmosférica (smog) conservación de los materiales artificiales (corrosión) y en el clima de un lugar. En la salud pública, por ejemplo, muestra una fuerte correlación estadística positiva entre la toxicidad del PM y las enfermedades respiratorias agudas²; siendo Lima, capital del Perú, una de las ciudades con frecuencia de asma en niños por la relación del MP proveniente de emisiones del parque automotor ${ }^{3}$. Por tal motivo, la caracterización química y vigilancia permanente del MP es básica e importante porque nos brinda información para la elaboración de estrategias y planes de control y mitigación del MP. 
En los Andes centrales del Perú se ubica la ciudad de Huancayo, que tiene una de las mayores poblaciones de la región andina peruana y donde las actividades económicas más importantes son: comercio, transporte e industria. Está ubicado en la margen izquierda del río Mantaro, en el extremo sur del valle del Mantaro; está dominado por un relieve plano con una baja gradiente altitudinal. Según el Instituto Nacional de Estadística e Informática del Perú (INEI) a nivel metropolitano (distritos de El Tambo, Huancayo y Chilca) tiene una población de 336 293 habitantes.

La ciudad de Huancayo está incluida en zonas de atención prioritaria de calidad del aire del Ministerio de Ambiente de Perú (MINAM); siendo el MP, el principal problema de calidad de aire $^{4}$. Por ello, este trabajo, por primera vez, caracteriza la composición química elemental,

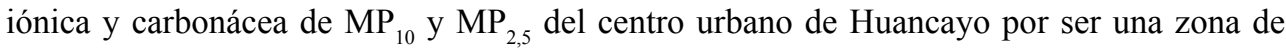
intensa actividad económica.

\section{PARTE EXPERIMENTAL}

\section{Ubicación de la estación de monitoreo}

La estación de monitoreo de MP se ubicó en el centro urbano de la ciudad de Huancayo $\left(12,07^{\circ} \mathrm{S} ; 75,21^{\circ} \mathrm{O} ; 3272 \mathrm{msnm}\right)$, cerca de la intersección de dos calles de gran confluencia vehicular y comercial (figura 1).

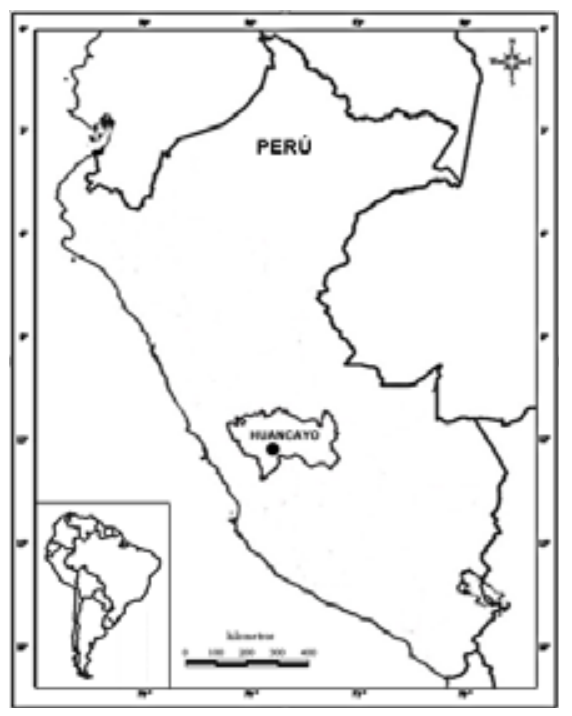

Figura 1. Ubicación del sitio de muestreo en el centro urbano de Huancayo, Perú. 


\section{Climatología}

La climatología de Huancayo fue obtenida de la estación del Huayao $\left(12,04^{\circ} \mathrm{S} ; 75,32^{\circ} \mathrm{O}\right.$; 3313 msnm) del Instituto Geofísico del Perú (IGP) desde 1966 hasta 20025 (figura 2). La variación de la temperatura describe dos períodos bien definidos durante el año, la primera de mínimos valores, centra $\neg$ dos en junio y julio (invierno), y la segunda de valores máximos entre octubre y diciembre (primavera). La temperatura media anual es de $11,9 \pm 1,2^{\circ} \mathrm{C}$.

Del mismo modo, la precipitación registra dos períodos bien definidos durante el año: época seca y húmeda. Entre junio y julio (invierno) se registran mínimas lluvias, mientras que entre enero y marzo (verano) se presentan las lluvias más intensas. Febrero es el mes con el mayor registro, alcanzando un valor acumulado de $129,1 \mathrm{~mm}$ de lluvia. El promedio acumulado anual de lluvia es de $752 \pm 44,3 \mathrm{~mm}$.

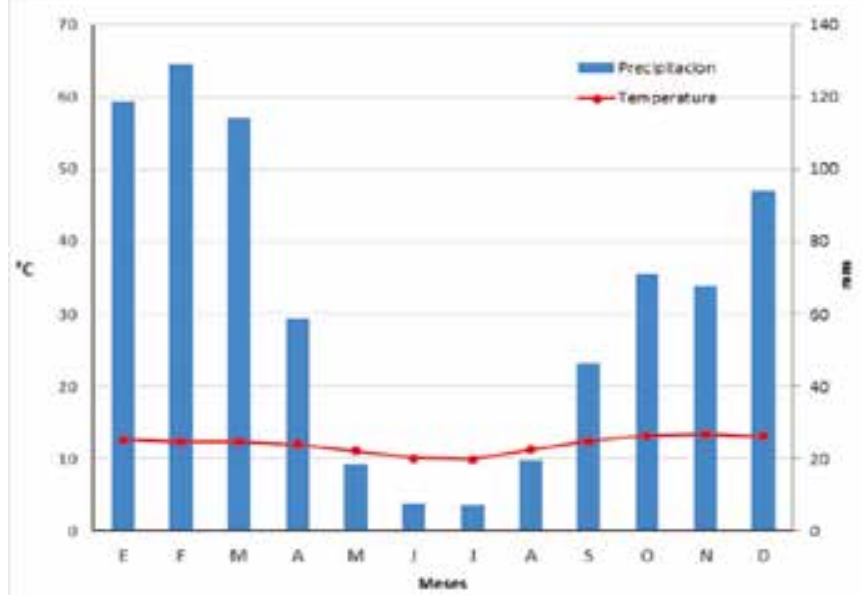

Figura 2. Climatograma de Huancayo en base a los años 1966-2002. Fuente: IGP

\section{Monitoreo}

El monitoreo del MP se realizó con un equipo colector de partículas de bajo volumen (PARTISOL FRM 2000 de Thermo Scientific, EE.UU.), el cual fue instalado a 10 metros del suelo. Complementariamente se instaló una estación meteorológica (modelo 232 de Weatherhawk, EE.UU.).

El monitoreo formó parte del programa de evaluación de calidad de aire de la zona alta y media de la cuenca hidrográfica del Mantaro de la Mesa de Diálogo Ambiental de la Región Junín (MEDIAREJ), por tal motivo, en Huancayo se monitoreó en cuatro meses: agosto del 2007, enero, abril y mayo del 2008. Durante este tiempo se realizaron colectas de $\mathrm{MP}_{10} \mathrm{y} \mathrm{MP}_{2,5}$ en filtros de teflón con diámetros de $47 \mathrm{~mm}$ y tamaño de poro de 2,0 $\mu \mathrm{m}$, para evaluar las concentraciones y composición química elemental de ambos tamaños de partículas y también se realizaron colectas adicionales de $\mathrm{MP}_{2.5}$ en filtros de cuarzo de 47 
$\mathrm{mm}$ de diámetro, previamente sometidos a $500^{\circ} \mathrm{C}$ por 2,5 horas para evitar que contengan fracciones carbonáceas. Esto se realizó para evaluar la concentración en el MP de iones de $\mathrm{NO}_{3}^{-}$y $\mathrm{SO}_{4}^{-}$y las especies carbonáceas: carbono orgánico (COrg) y carbono elemental (CE). La frecuencia de monitoreo del MP10 fue de cada tres días y del MP2,5 fue de cada 15 días. La colecta de ambos tamaños de partículas se realizó con un flujo constante de 16,7 L/ min por 24 horas según lo establecido en el Decreto Supremo Nº74-2001 PCM y D.S. N ${ }^{\circ}$ 003-2008-MINAM de la normativa peruana.

\section{Análisis de muestras}

Las muestras colectadas de $\mathrm{MP}_{10}$ y $^{\mathrm{MP}_{2.5}}$ fueron enviadas a EE.UU. para ser analizadas por el Laboratorio CHESTER LabNet (http://www.chesterlabnet.com) acreditado por el NELAC (National Environmental Laboratory Acreditation Conference) de EE.UU.

Las concentraciones de $\mathrm{MP}_{10} \mathrm{y} \mathrm{MP}_{2,5}$ se determinaron por el método gravimétrico establecido en el Método de la EPA IO-3.1 a través de la diferencia del peso inicial y final de cada filtro mediante una microbalanza de $\pm 0,010 \mathrm{mg}$ de precisión.

La composición química elemental del MP se obtuvo mediante el análisis de hasta 38 elementos posibles a detectar a través de la técnica de la fluorescencia de rayos X (XRF) establecido en el método analítico EPA IO-3.3. Los límites de detección de los elementos analizados en el MP se muestran en la tabla 1.

Tabla 1. Límites de detección de los elementos analizados en el material particulado.

\begin{tabular}{cccccc}
\hline Elemento & $\boldsymbol{\mu g}$ /filtro & Elemento & $\boldsymbol{\mu g} /$ filtro & Elemento & $\boldsymbol{\mu g}$ /filtro \\
\hline $\mathrm{Al}$ & 0,136 & $\mathrm{Sr}$ & 0,038 & $\mathrm{Ag}$ & 0,129 \\
$\mathrm{Sb}$ & 0,185 & $\mathrm{P}$ & 0,081 & $\mathrm{~Pb}$ & 0,067 \\
$\mathrm{As}$ & 0,029 & $\mathrm{Ga}$ & 0,036 & $\mathrm{~K}$ & 0,051 \\
$\mathrm{~S}$ & 0,067 & $\mathrm{Ge}$ & 0,022 & $\mathrm{Rb}$ & 0,024 \\
$\mathrm{Ba}$ & 1,424 & $\mathrm{Fe}$ & 0,014 & $\mathrm{Se}$ & 0,024 \\
$\mathrm{Br}$ & 0,022 & $\mathrm{In}$ & 0,336 & $\mathrm{Si}$ & 0,095 \\
$\mathrm{Cd}$ & 0,132 & $\mathrm{La}$ & 0,870 & $\mathrm{Na}$ & 2,543 \\
$\mathrm{Ca}$ & 0,034 & $\mathrm{Mg}$ & 0,305 & $\mathrm{Ti}$ & 0,024 \\
$\mathrm{Cl}$ & 0,082 & $\mathrm{Mn}$ & 0,026 & $\mathrm{~V}$ & 0,017 \\
$\mathrm{Co}$ & 0,014 & $\mathrm{Hg}$ & 0,060 & $\mathrm{Y}$ & 0,036 \\
$\mathrm{Cu}$ & 0,014 & $\mathrm{Mo}$ & 0,060 & $\mathrm{Zn}$ & 0,017 \\
$\mathrm{Cr}$ & 0,017 & $\mathrm{Ni}$ & 0,014 & $\mathrm{Zr}$ & 0,043 \\
$\mathrm{Sn}$ & 0,231 & $\mathrm{Pd}$ & 0,122 & & \\
\hline
\end{tabular}

Fuente: CHESTER LabNet

Los iones $\mathrm{NO}_{3}^{-}$y $\mathrm{SO}_{4}{ }^{\circ}$ se analizaron por la técnica de cromatografía con un equipo Dionex ICS-3000 (Thermo Fisher Scientific, EE.UU.). Estos fueron separados en una columna de intercambio iónico a alta presión y extraídos a partir de sustratos de filtro por inmersión en $10,0 \mathrm{~mL}$ de agua desionizada en 60 minutos con sonicación. 
Las especies carbonáceas de COrg y CE se analizaron utilizando el método térmico/óptico basado en el método NIOSH 5040. El COrg se definió como todo el carbono producido en una única fase móvil de gas helio, y el CE como todo el carbono producido en una fase móvil de la mezcla del gas helio/oxígeno.

\begin{abstract}
Análisis de datos
Con la información meteorológica de dirección y velocidad de viento se elaboraron rosas de vientos con el programa WRPlot View v8.0 (Lakes Environmental Software, EE.UU.). Se utilizó la hoja de cálculo Excel de Microsoft Office para la conversión de datos reportados del laboratorio de microgramos por filtro ( $\mu \mathrm{g} /$ filtro) a microgramos por metro cúbico $(\mu \mathrm{g} /$ $\mathrm{m}^{3}$ ), tomando en cuenta la temperatura y presión atmosférica del sitio de monitoreo por el Método EPA IO-2.4. Con el programa R (www.r-project.org) se calculó los estadísticos descriptivos para cada tamaño de materia particulado y componente químico analizado, para luego compararlas con la normativa peruana vigente.
\end{abstract}

También se realizó un análisis de la variabilidad de las concentraciones diarias de $\mathrm{MP}_{10} \mathrm{y}$ $\mathrm{MP}_{2,5}$ por período seco (agosto y mayo) y lluvioso (enero y abril) ${ }^{5}$. Finalmente, se calcularon los porcentajes de los elementos químicos, iones y especies carbonáceas detectados en el

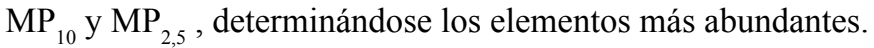

\title{
RESULTADOS Y DISCUSIÓN
}

Como se observa en la figura 3, entre agosto 2007 y mayo 2008, periodo seco, los vientos predominantes fueron en dirección suroeste y noreste con una velocidad frecuente de 2,6 a $4,1 \mathrm{~m} / \mathrm{s}$; en cambio en enero y abril del 2008 , periodo lluvioso, los vientos predominantes fueron con dirección hacia el sur y sursuroeste con velocidades dominantes de 4,1 a 5,7 $\mathrm{m} / \mathrm{s}$. En ambos periodos las velocidades máximas fueron mayores a 10,3 m/s. Por lo que la dirección vientos varía según periodo lluvioso y seco.
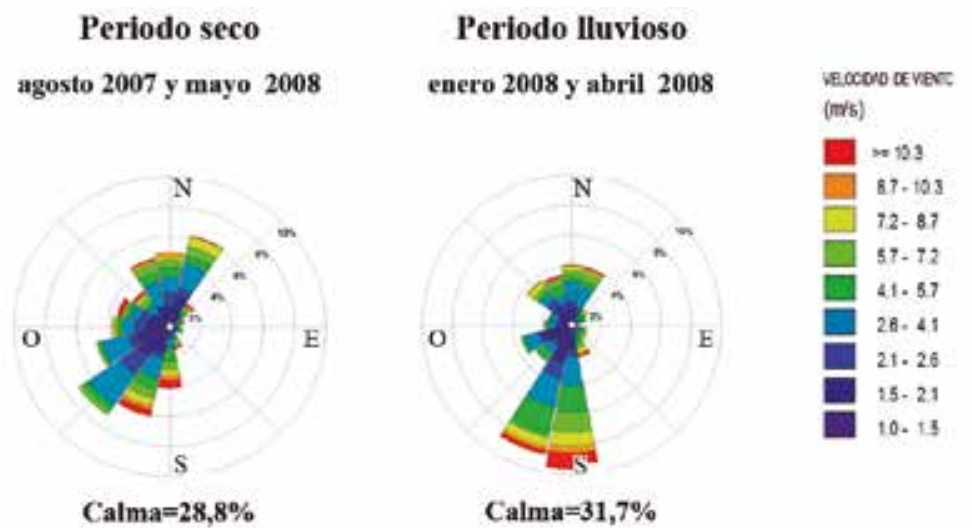

Figura 3. Rosas de vientos obtenidas en la estación de monitoreo del centro urbano de Huancayo en periodo seco (agosto 2007, mayo 2008) y lluvioso (enero 2008, abril 2008). 


\section{Composición elemental}

\section{Material particulado menor a $10 \mu \mathrm{m}\left(\mathrm{MP}_{10}\right)$ :}

Como se aprecia en la tabla 2 el promedio para el $\mathrm{MP}_{10}$ en el centro de Huancayo para los cuatro meses de evaluación fue de $64,54 \pm 30,87 \mu \mathrm{g} / \mathrm{m}^{3}$ superando el Estándar de Calidad Ambiental (ECA) anual $\left(50 \mu \mathrm{g} / \mathrm{m}^{3}\right)$ en más del $29 \%$. Siendo el valor máximo diario registrado de $143 \mu \mathrm{g} / \mathrm{m}^{3}$. Ningún valor diario de $\mathrm{MP}_{10}$ superó el ECA de 24 horas peruano $\left(150 \mu \mathrm{g} / \mathrm{m}^{3}\right)$.

Comparando el valor promedio de $\mathrm{MP}_{10}$ de Huancayo, muestra menor concentración con respecto al distrito de Ate-Lima-Perú $\left(137,37 \mu \mathrm{g} / \mathrm{m}^{3} ; 355 \mathrm{msnm}\right)^{6}$ y otras ciudades de América Latina como Santiago-Chile (69 $\left.\mu \mathrm{g} / \mathrm{m}^{3} ; 550 \mathrm{msnm} ; 2007\right)$; y es ligeramente mayor que $\mathrm{La}$ Paz-Bolivia (54 $\left.\mu \mathrm{g} / \mathrm{m}^{3} ; 3640 \mathrm{msnm} ; 2007\right)^{7}$.

Respecto a la composición elemental los metales ( $\mathrm{Ca}, \mathrm{Al}, \mathrm{Fe}, \mathrm{K}, \mathrm{Mg}, \mathrm{Ti}, \mathrm{Na}, \mathrm{Zn}, \mathrm{Mn}, \mathrm{Pb}$, $\mathrm{Cu}, \mathrm{Sr}, \mathrm{Rb}, \mathrm{Cr}, \mathrm{Cd}, \mathrm{Ni}, \mathrm{V}, \mathrm{Zr}$ y Y) constituyeron aproximadamente $11,9 \%$ del peso del $\mathrm{PM}_{10}$, los metaloides ( $\mathrm{Si}$, As y Sb) el 7,5\% del peso del $\mathrm{PM}_{10}$, y los no metales ( $\mathrm{S}, \mathrm{Cl}, \mathrm{P}, \mathrm{Br}$ y $\mathrm{Se}$ ) el 1,9\% del peso del PM10. Los cinco elementos más abundantes del MP10 de Huancayo, durante el periodo de monitoreo, fueron el $\mathrm{Si}, \mathrm{Ca}, \mathrm{Al}, \mathrm{Fe}$ y $\mathrm{K}$ ocupando el 7,6\% del $\mathrm{MP}_{10}$, indicando la dominancia de elementos geológicos cuya fuente principal es el suelo ${ }^{8}$.

Tabla 2. Media aritmética, desviación estándar (s), máximo valor (máx.) y número de datos (n) para el $\mathrm{MP}_{10}\left(\right.$ en $\left.\mu \mathrm{g} / \mathrm{m}^{3}\right)$ del centro urbano de Huancayo en agosto 2007 y enero, abril y mayo del 2008.

\begin{tabular}{cccccccccc}
\hline Variable & media & s & máx. & n & Variable & Media & s & máx. & n \\
\hline $\mathbf{M P}_{\mathbf{1 0}}$ & 64,536 & 30,87 & 143,003 & 41 & $\mathbf{A s}$ & 0,022 & 0,03 & 0,118 & 38 \\
$\mathbf{S i}$ & 4,796 & 2,42 & 10,984 & 40 & $\mathbf{S b}$ & 0,021 & 0,01 & 0,029 & 9 \\
$\mathbf{C a}$ & 2,448 & 1,25 & 5,803 & 41 & $\mathbf{P}$ & 0,019 & 0,01 & 0,048 & 25 \\
$\mathbf{A l}$ & 1,986 & 1,07 & 4,652 & 40 & $\mathbf{C u}$ & 0,009 & 0,01 & 0,024 & 40 \\
$\mathbf{F e}$ & 1,425 & 0,73 & 3,231 & 40 & $\mathbf{C d}$ & 0,008 & 0,0004 & 0,008 & 3 \\
$\mathbf{K}$ & 1,083 & 0,61 & 3,145 & 40 & $\mathbf{S r}$ & 0,008 & 0,004 & 0,024 & 39 \\
$\mathbf{S}$ & 0,882 & 0,55 & 2,227 & 40 & $\mathbf{Z r}$ & 0,005 & 0,002 & 0,008 & 26 \\
$\mathbf{C l}$ & 0,348 & 0,29 & 1,340 & 40 & $\mathbf{V}$ & 0,005 & 0,003 & 0,012 & 16 \\
$\mathbf{N a}$ & 0,249 & 0,10 & 0,427 & 9 & $\mathbf{R b}$ & 0,004 & 0,002 & 0,009 & 39 \\
$\mathbf{M g}$ & 0,186 & 0,11 & 0,474 & 38 & $\mathbf{B r}$ & 0,003 & 0,002 & 0,010 & 35 \\
$\mathbf{T i}$ & 0,131 & 0,07 & 0,304 & 40 & $\mathbf{C r}$ & 0,003 & 0,002 & 0,008 & 32 \\
$\mathbf{Z n}$ & 0,057 & 0,03 & 0,122 & 40 & $\mathbf{Y}$ & 0,003 & $\ldots$ & 0,003 & 1 \\
$\mathbf{M n}$ & 0,040 & 0,02 & 0,096 & 40 & $\mathbf{N i}$ & 0,002 & 0,002 & 0,008 & 15 \\
$\mathbf{P b}$ & 0,035 & 0,03 & 0,190 & 40 & $\mathbf{S e}$ & 0,002 & 0,0001 & 0,002 & 2 \\
\hline
\end{tabular}

Dada la variabilidad climática de la zona, luego de determinar la normalidad de los datos de $\mathrm{MP}_{10}$ con la prueba de Shapiro-Wilk ( $\mathrm{p}$-value $\left.=0,09\right)$, diferencia de varianzas $(\mathrm{F}=0,311$; $\mathrm{p}$-value $=0,013)$ y de someter la prueba $\mathrm{T}$ para varianzas desiguales $(\mathrm{t}=-3,21 ; \mathrm{p}$-value $<0,01)$ se concluyó que sí hay diferencia significativa entre las concentraciones de $\mathrm{MP}_{10}$ 
registrados entre el periodo seco y lluvioso. Por lo que las concentraciones del MP10 en los meses secos $\left(77,31 \pm 34,95 \mu \mathrm{g} / \mathrm{m}^{3}\right)$ es mayor que los meses lluviosos $\left(50,40 \pm 19,08 \mu \mathrm{g} / \mathrm{m}^{3}\right)$. Esta variabilidad diaria del $\mathrm{MP}_{10}$ según periodos secos y húmedos se muestra en la figura 4.

\section{Material particulado menor a 2,5 $\mu \mathrm{m}\left(\mathrm{MP}_{2,5}\right)$}

Como se puede apreciar en la tabla 3, la media aritmética del $\mathrm{MP}_{2.5}$ para Huancayo fue de $34,47 \pm 14,75 \mu \mathrm{g} / \mathrm{m}^{3}$ superando en más del doble el ECA anual $\left(15 \mu \mathrm{g} / \mathrm{m}^{3}\right)$. Sólo un valor diario $\left(67,79 \mu \mathrm{g} / \mathrm{m}^{3}\right)$ registrado el 25 de agosto del 2007 superó el ECA para 24 horas (50 $\left.\mu \mathrm{g} / \mathrm{m}^{3}\right)$. Comparando el promedio anual del $\mathrm{MP}_{2,5}$ obtenido en Huancayo con otras ciudades, registra menores concentraciones con respecto a Bogotá - Colombia $\left(35,1 \mu \mathrm{g} / \mathrm{m}^{3}\right)^{9}$; pero mayores concentraciones en comparación con Lima $\left(31,5 \mu \mathrm{g} / \mathrm{m}^{3}\right)$; Santiago $\left(26,1 \mu \mathrm{g} / \mathrm{m}^{3}\right)$; y México D.F-México $\left(26,2 \mu \mathrm{g} / \mathrm{m}^{3}\right)^{9}$. Cabe resaltar que el $\mathrm{MP}_{2,5}$ es el tamaño de partícula de mayor importancia en la problemática de calidad de aire y salud pública en Huancayo.

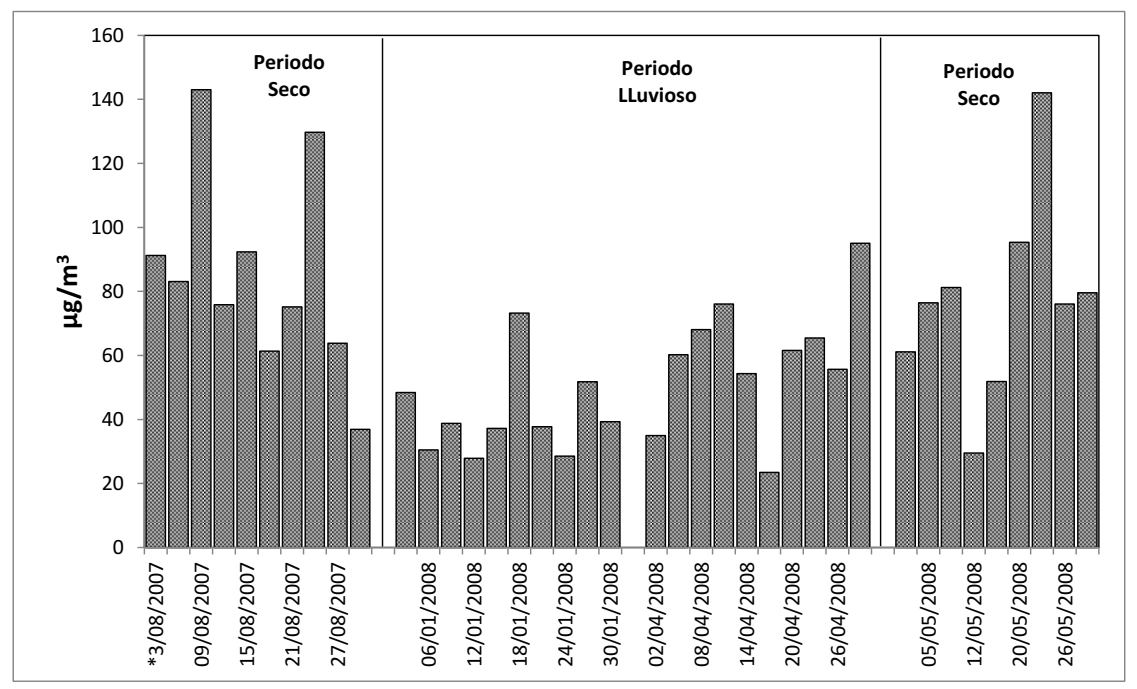

Figura 4. Variación diaria del $\mathrm{MP}_{10}$ en el centro urbano de Huancayo durante los meses de agosto 2007, enero, abril y mayo del 2008.

Los cinco elementos químicos más abundantes en el $\mathrm{PM}_{2,5}$ fueron el $\mathrm{S}, \mathrm{Si}, \mathrm{K}, \mathrm{Ca}$ y $\mathrm{Cl}$; la gran presencia del $\mathrm{S}$ en este tamaño de partícula podría estar relacionado con la quema de biomasa $^{10}$ y la combustión del diésel ${ }^{11}$, debido a que cerca al lugar de monitoreo existen hornos de pollería y parrilleras donde usan carbón y leña como combustible. Asimismo, existe gran tránsito vehicular. Los elementos $\mathrm{Si}, \mathrm{K}$ y Ca pertenecen a los elementos geológicos de los suelos ${ }^{8}$.

Los metales en el peso del $\mathrm{MP}_{2,5}$ constituyeron aproximadamente el 4,1\%, los metaloides representaron el 2,1\% y los no metales representaron el 3,8\%. Si comparamos estos porcentajes con los registrados en el $\mathrm{MP}_{10}$ se aprecia una reducción en aproximadamente tres veces de 
la proporción de los metales y metaloides, por lo que estos elementos se encontrarían en las fracciones gruesas $\left(\mathrm{MP}_{10}\right)$. Caso contrario se observa para los no metales, predominando en la fracción fina $\left(\mathrm{MP}_{2,5}\right)$.

Tabla 3. Media aritmética, desviación estándar (s), máximo valor (máx.) y número de datos (n) para el $\mathrm{MP}_{2,5},\left(\right.$ en $\left.\mu \mathrm{g} / \mathrm{m}^{3}\right)$ del centro urbano de Huancayo en agosto 2007 y enero, abril y mayo del 2008.

\begin{tabular}{cccccccccc}
\hline Variable & media & $\mathbf{S}$ & máx. & $\mathbf{n}$ & Variable & media & $\mathbf{S}$ & máx. & $\mathbf{~}$ \\
\hline $\mathbf{M P}_{2,5}$ & 34,465 & 14,75 & 67,792 & 9 & $\mathbf{P b}$ & 0,018 & 0,01 & 0,043 & 9 \\
$\mathbf{S}$ & 0,946 & 0,69 & 1,929 & 9 & $\mathbf{T i}$ & 0,017 & 0,01 & 0,027 & 9 \\
$\mathbf{S i}$ & 0,684 & 0,31 & 1,129 & 9 & $\mathbf{A s}$ & 0,014 & 0,01 & 0,033 & 9 \\
$\mathbf{K}$ & 0,418 & 0,25 & 1,002 & 9 & $\mathbf{P}$ & 0,009 & $\ldots$ & 0,009 & 1 \\
$\mathbf{C a}$ & 0,346 & 0,13 & 0,501 & 9 & $\mathbf{M n}$ & 0,005 & 0,002 & 0,009 & 9 \\
$\mathbf{C l}$ & 0,335 & 0,16 & 0,497 & 8 & $\mathbf{C u}$ & 0,004 & 0,005 & 0,015 & 8 \\
$\mathbf{A l}$ & 0,334 & 0,18 & 0,618 & 9 & $\mathbf{V}$ & 0,003 & $\ldots$ & 0,003 & 1 \\
$\mathbf{F e}$ & 0,208 & 0,09 & 0,330 & 9 & $\mathbf{B r}$ & 0,003 & 0,001 & 0,005 & 8 \\
$\mathbf{M g}$ & 0,043 & 0,01 & 0,051 & 4 & $\mathbf{N i}$ & 0,003 & 0,002 & 0,004 & 2 \\
$\mathbf{S b}$ & 0,020 & $\ldots$ & 0,020 & 1 & $\mathbf{S r}$ & 0,002 & $\ldots$ & 0,002 & 1 \\
$\mathbf{Z n}$ & 0,018 & 0,01 & 0,032 & 9 & & & & & \\
\hline
\end{tabular}

Al igual que el caso del $\mathrm{MP}_{2,5}$ se realizó la prueba de normalidad de Shapiro-Wilk (p-value = $0,002)$, pero los datos no cumplieron normalidad. Por ello, se aplicó la prueba no paramétrica de Mann Whitney ( $\mathrm{p}$-value $=0,037$ ), la que indicó que también existe una diferencia significativa entre los dos períodos.

La media aritmética en periodo seco fue de 42,61 $\pm 14,13 \mu \mathrm{g} / \mathrm{m}^{3}$ siendo mayor al registrado en el periodo lluvioso $24,28 \pm 8,07 \mu \mathrm{g} / \mathrm{m}^{3}$. Para evaluar diferencias significativas entre estación seca y húmeda primero En la figura 5 se puede apreciar la variación diaria de las concentraciones del MP2,5 en el zona urbana de Huancayo agrupado por estaciones.

\section{Iones}

Como se observa en la tabla 5, en el $\mathrm{MP}_{2,5}$ existe mayor concentración y variabilidad del $\mathrm{SO}_{4}=$ en comparación con el $\mathrm{NO}_{3}^{-}$. La mayor presencia de $\mathrm{SO}_{4}{ }^{-}$, se debería al parque automotor, pues el centro urbano es muy transitado por vehículos menores y transporte público, lo que estaría aumentando las concentraciones de $\mathrm{SO}_{4}{ }^{-}$por procesos de combustión, típicas en áreas urbanas ${ }^{11}$. 


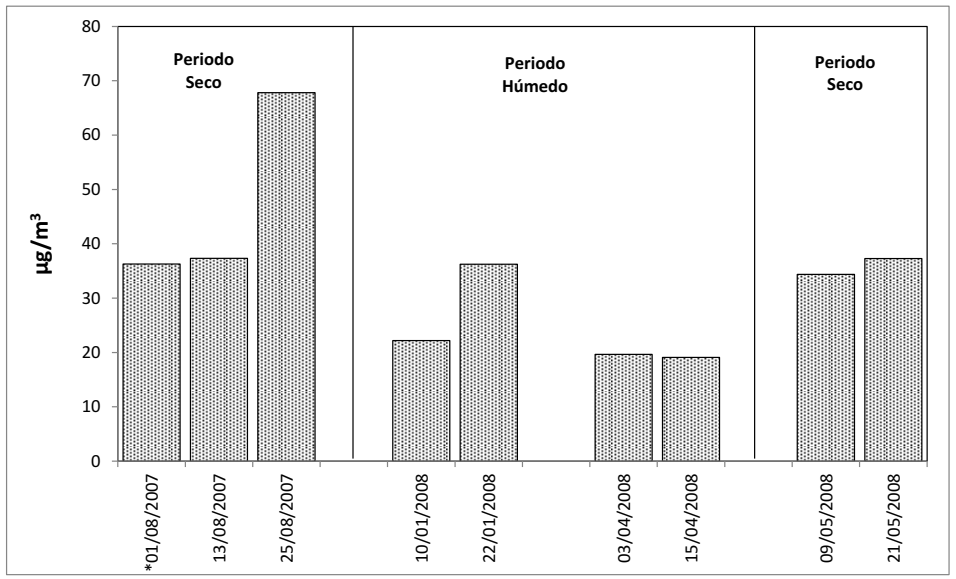

Figura 5. Concentración del $\mathrm{MP}_{2,5}$ en el centro urbano de Huancayo durante agosto 2007, enero, abril y mayo del 2008.

En la figura 6 se muestra la variación diaria de las concentraciones de $\mathrm{SO}_{4}{ }^{-} \mathrm{y} \mathrm{NO}_{3}^{-}$en el $\mathrm{MP}_{2,5}$. El promedio registrado de Huancayo para $\mathrm{NO}_{3}^{-}$fue de $1,03 \pm 0,52 \mu \mathrm{g} / \mathrm{m}^{3} \mathrm{y} \mathrm{SO}_{4}{ }^{-}$fue de $2,45 \pm 1,77 \mu \mathrm{g} / \mathrm{m}^{3}$, en comparación con lo registrado en Sao Paulo- Brasil $\left(\mathrm{NO}_{3}{ }^{-}=2,1 \mu \mathrm{g} / \mathrm{m}^{3}\right.$; $\left.\mathrm{SO}_{4}=3,3 \mu \mathrm{g} / \mathrm{m}^{3}\right)^{12}$, y el área urbana de Guangzhou-China ${ }^{13}\left(\mathrm{NO}_{3}{ }^{-}=8,8 \mu \mathrm{g} / \mathrm{m}^{3} ; \mathrm{SO}_{4}=38,6\right.$ $\left.\mu \mathrm{g} / \mathrm{m}^{3}\right)$ fue menor. Mientras que comparado con la ciudad de Piracicaba-Brasil $\left(\mathrm{NO}_{3}{ }^{-}=0,5\right.$ $\left.\mu \mathrm{g} / \mathrm{m}^{3} ; \mathrm{SO}_{4}{ }^{=}=1,8 \mu \mathrm{g} / \mathrm{m}^{3}\right)^{12}$, Huancayo registra mayores valores.

Tabla 4. Media aritmética, desviación estándar (s), número de datos (n) de los sulfatos y nitratos contenidos en el $\mathrm{MP}_{2,5}$, expresado en $\mu \mathrm{g} / \mathrm{m}^{3}$, en el centro urbano de Huancayo.

\begin{tabular}{lccc}
\hline Var. & media & $\mathbf{s}$ & $\mathbf{n}$ \\
\hline $\mathrm{NO}_{3}{ }^{-}$ & 1,03 & 0,52 & 6 \\
$\mathrm{SO}_{4}{ }^{-}$ & 2,45 & 1,77 & 7 \\
\hline
\end{tabular}

\section{Especies carbonáceas}

En la tabla 5 se observa que existe mayor cantidad de carbono orgánico (COrg) respecto al carbono elemental $(\mathrm{CE})$ en el $\mathrm{PM}_{2,5}$. La figura 6 muestra la variabilidad diaria registrada en este estudio.

Tabla 5. Media aritmética, desviación estándar (s), número de datos (n) del carbono orgánico (COrg) y carbono elemental (CE) contenidos en el $\mathrm{PM}_{2,5}$, expresado en $\mu \mathrm{g} / \mathrm{m}^{3}$, en el centro urbano de Huancayo.

\begin{tabular}{cccc}
\hline Var. & media & $\mathbf{s}$ & $\mathbf{n}$ \\
\hline & & & \\
\cline { 2 - 4 } COrg & 8,284 & 4,733 & 7 \\
CE & 7,062 & 1,940 & 6 \\
\hline
\end{tabular}



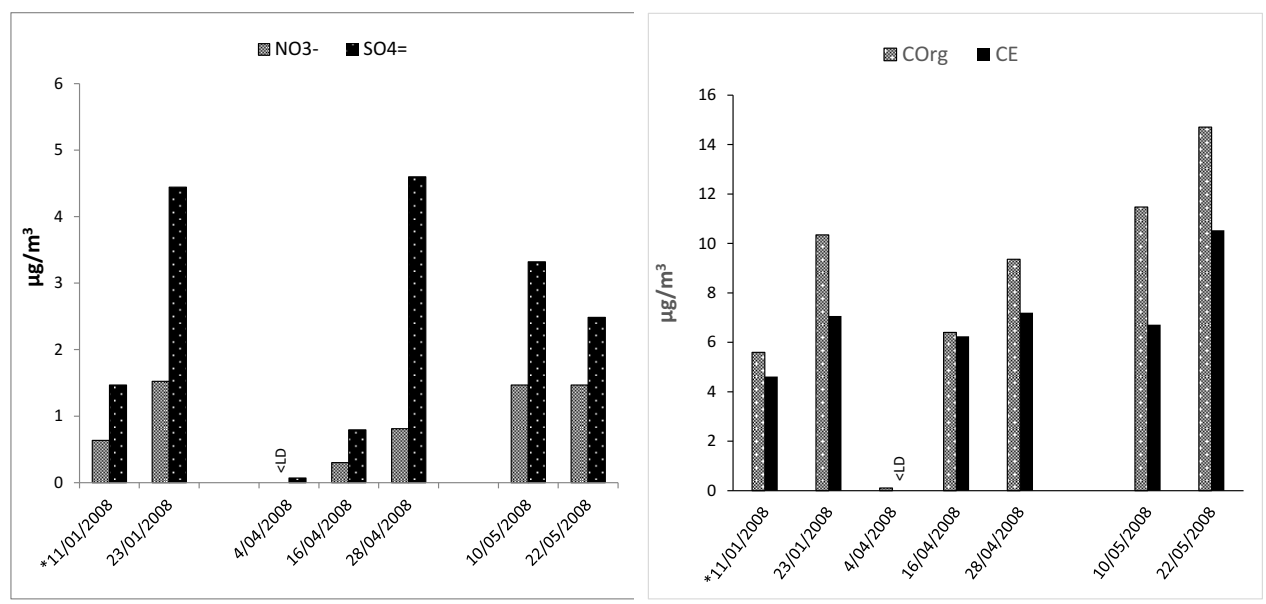

Figura 6. Concentraciones registradas de $\mathrm{NO}^{3}-\mathrm{y} \mathrm{SO}_{4}=$, COrg y $\mathrm{CE}$ en $\mathrm{MP}_{2,5}$ en el centro urbano de Huancayo durante los meses de agosto 2007, enero, abril y mayo del 2008

(LD: Límite de Detección)

Para evaluar la dependencia de ambas especies carbonáceas se realizó la prueba de correlación de Spearman, para comprobar la hipótesis de regresión lineal significativa entre los dos parámetros evaluados, dando un coeficiente de correlación alto $(\mathrm{r}=0,903 ; \mathrm{p}$-value $=0,005)$, por lo que se puede decir que estas dos variables provienen de una misma fuente. Sabiendo que el CE se produce principalmente de los vehículos que funcionan a base de combustibles fósiles, grandes emisiones de compuestos carbonaceos ${ }^{14} \mathrm{y}$ dada la gran confluencia vehicular en la zona de muestreo de material particulado, podemos afirmar que el COrg y el CE, registrado para este estudio en la ciudad de Huancayo, provienen principalmente del parque automotor.

\section{Componentes del material particulado}

A manera de resumen, se elaboraron los gráficos de la figura 7 con el fin de representar las proporciones que ocupan los componentes analizados en el MP del centro de Huancayo. En caso del $\mathrm{MP}_{10}$, a nivel elemental el $12 \%$ estuvo representado por los metales, $7 \%$ de metaloides, $2 \%$ de no metales. Para el $\mathrm{MP}_{2,5}$ las fracciones carbonáceas: COrg y CE representaron el $24 \%$ y $20 \%$, respectivamente, mientras que a nivel elemental: el $4 \%$ estuvo representado por los metales, $2 \%$ de metaloides y $4 \%$ de no metales; y las fracciones iónicas: $\mathrm{NO}_{3}^{-} \mathrm{y} \mathrm{SO}_{4}{ }^{-}$representaron el $4 \%$ y $7 \%$, respectivamente. 

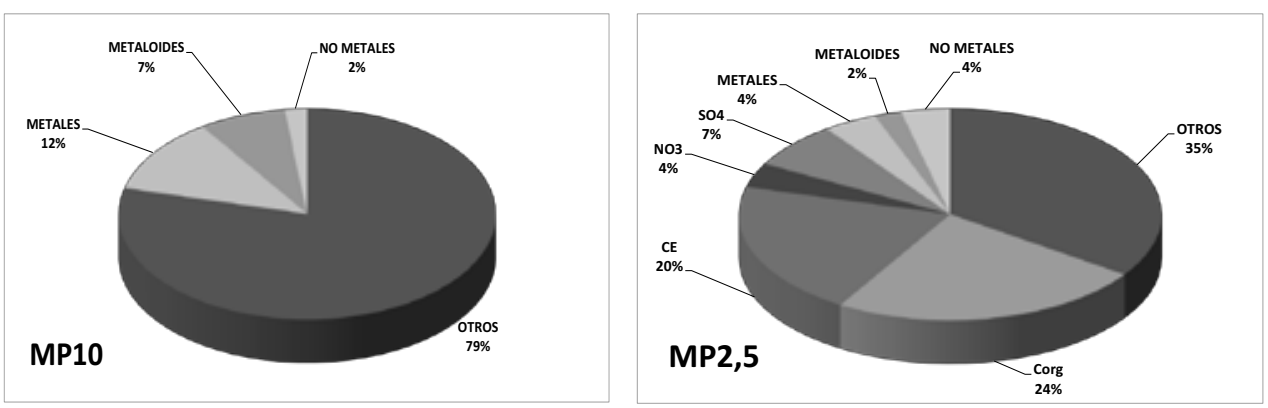

Figura 7. Porcentajes de los componentes químicos analizados en el $\mathrm{MP}_{10}$ y $\mathrm{MP}_{2,5}$ del centro de Huancayo.

\section{CONCLUSIONES}

El $\mathrm{MP}_{10} \mathrm{y} \mathrm{MP}_{2,5}$ en los cuatro meses de monitoreo registraron valores mayores de los ECAs anuales peruanos. Siendo el $\mathrm{MP}_{2,5}$ que registró valores de más del doble del ECA anual. Debido a su tamaño, el $\mathrm{MP}_{2,5}$ es de mayor importancia en la problemática de calidad de aire, considerándose un agente de riesgo potencial a la salud de los pobladores de Huancayo.

Existe mayor concentración de $\mathrm{MP}_{10} \mathrm{y} \mathrm{MP}_{2,5}$ en periodos secos, lo que nos estaría indicando su relación con los parámetros climáticos.

En el $\mathrm{MP}_{10}$ se muestra una apreciable cantidad de metales (12\%) y abundancia de elementos geológicos. En cambio en el $\mathrm{MP}_{2,5}$ se ha registrado mayoritariamente el $\mathrm{S}$, no metales y concentraciones importantes de $\mathrm{COrg}, \mathrm{CE} \mathrm{y} \mathrm{SO}_{4}{ }^{=}$las cuales estarían siendo atribuidas al parque automotor.

\section{AGRADECIMIENTO}

Al Arzobispado de Huancayo y Mesa de Diálogo Ambiental de la Región Junín y a su proyecto: Fortalecimiento de la Gestión Ambiental para la lucha contra la contaminación de la zona alta-media de la cuenca del río Mantaro-Junín conocido como "El Mantaro Revive" el cual fue financiado por el Fondo Ítalo Peruano - FIP por la data otorgada en este estudio. Al fondo Innóvate-Perú por el apoyo a través de contrato 324-PNICP-PIAP-2015 de la Universidad Nacional del Centro del Perú. A la United States Agency International for Development (USAID) vía el proyecto: "Impacto del transporte transfronterizo de contaminantes del aire sobre los Andes Centrales de Perú relacionados a la quema de vegetación en la Amazonía" del programa PEER Sciences. 


\section{BIBLIOGRAFÍA}

1. Pöschl U. Atmospheric Aerosols: Composition, Transformation, Climate and Health Effects. Angew Chem Int Ed Engl. 2005; 44: 7520 - 7540.

2. Anderson JO, Josef G, Stolbach A. Clearing the Air: A Review of the Effects of Particulate Matter Air Pollution on Human Health. J Med Toxicol. 2012; 8(2): 166-175.

3. Carbajal AL, Barraza VA, Durand PR, MH, Espinoza LR, Chiarella OP, et al. Impac of traffic flow on thea asthma prevalence among school children in Lima, Peru. J Asthma. 2007; 44(3): 197-202.

4. Consejo Nacional del Ambiente (CONAM). Plan "A Limpiar el Aire de Huancayo". Decreto del Consejo Directivo N 029-2005-CONAM/CD.

5. Instituto Geofísico del Perú (IGP). Atlas Climático de precipitación y temperatura del aire en la cuenca del río Mantaro. Volumen I. Lima: Fondo Editorial CONAM; 2005.

6. Servicio Nacional de Meteorología e Hidrología (SENAMHI). Evaluación de la calidad del aire en Lima Metropolitana 2011. Lima: Dirección General de Investigación y Asuntos Ambientales del Servicio Nacional de Meteorología e Hidrología; 2011.

7. Programa de las Naciones para el Medio Ambiente - PNUMA. Perspectivas del Medio Ambiente: América Latina y el Caribe Geo ALC 3 [Internet]. 2010 [citado el 12 de octubre del 2016] Disponible en: https://wedocs.unep.org/rest/bitstreams/13194/retrieve

8. Artaxo P, Oyola P, Martínez R. Aerosol composition and source apportionment in Santiago de Chile. Nucl Instrum Methods Phys Res B. 1999; 150 (1-4): 409-416.

9. Clean Air Institute. La calidad del Aire en América Latina: Una visión Panorámica [Internet]. Washington D.C. [citado en 22 de marzo de 2013]. Disponible en: http:// www.cleanairinstitute.org/calidaddelaireamericalatina/cai-report-spanish.pdf.

10. Echalar F, Artaxo P, Vanderlei J, Yamasoe M, Gerab F. Long-term monitoring of atmospheric aerosols in the Amazon Basin: Source identification and apportionment. $\mathrm{J}$ Geophys Res. 1998; 103 (D24): 31849-31864.

11. Stechmann H, Dannecker W. Characterization and source analysis of vehicle generated aerosols. J Aerosol Sci. 1990; 21(1): S287-S290.

12. Souza DZ, Vasconcellos PC, Lee H, Aurela M, Saarnio K, Teinilä K, Hillamo R. Composition of PM2.5 and PM10 Collected at Urban Sites in Brazil. Aerosol Air Qual Res. 2014; 14: 168-176.

13. Chang S, Chou C, Liu S, Zhang Y. The Characteristics of PM2.5 and Its Chemical Compositions between Different Prevailing Wind Patterns in Guangzhou. Aerosol Air Qual Res. 2013; 13: 1373-1383.

14. Kwangsam N, Sawan AA, Song Ch, Cocker DR. Primary and secondary carbonaceous species in the atmosphere of Western Riverside County, California. Atmos Environ. 2004; 38: 1345-1355. 